\title{
Surface Alloying of Titanium Using a Nanosecond Laser with a Light-Transmitting Resin
}

\author{
Takuto Yamaguchi and Hideki Hagino \\ Osaka Research Institute of Industrial Science and Technology, \\ 2-7-1, Ayumino, Izumi, Osaka 594-1157, Japan \\ E-mail: t_yamaguchi@tri-osaka.jp
}

\begin{abstract}
Titanium and its alloys have various excellent properties such as good corrosion resistance and high specific strength. Yet they also have poor tribological properties that preclude their application to sliding parts. Laser alloying is an effective process for improving surface properties such as wear resistance and has numerous advantages over conventional surface modification techniques. The process has an important flaw, however, as defects such as cracks or voids tend to appear in the laser-alloyed zone. Earlier our group developed a novel laser alloying technique using a lighttransmitting resin as a source for the carbon element. The technique produces a crack-free laseralloyed zone. In the present study we applied a nanosecond pulsed laser for the laser alloying treatment in an attempt to improve the process stability by minimizing the mass of the molten metal. The defect free laser-alloyed zone with a smooth surface was obtained. High volume fractions of titanium oxycarbide particles were dispersed in the laser-alloyed zone. The laser-alloyed zone had a low friction coefficient against the WC-Co ball and superior wear resistance.
\end{abstract}

DOI: 10.2961/jlmn.2017.03.0002

Keywords: laser alloying, titanium, light-transmitting resin, surface modification, wear resistance

\section{Introduction}

Titanium and its alloys have various excellent properties such as good corrosion resistance and high specific strength. However, they have not been applied to the sliding parts under sever wear condition due to their poor tribological properties [1]. Several surface-hardening treatments have been applied to improve the wear resistance of titanium. In applications with hard coatings such as PVD and CVD, poor adhesion strength between the coating layer and base metal often results in delamination [2]. In plasma carburizing treatments, the long treatment time at high temperature tends to coarsen the grain and cause heat distortion [3].

Laser surface alloying, an effective process for improving wear resistance, is performed by melting the surface of a substrate with added materials, mixing the components together, and rapidly solidifying the mixture [4]. The process attains the advantages of low thermal strain, fine microstructure, and flexibility in the choice of substrates and added materials [5]. Laser alloying can also be applied selectively to circumscribed areas of a treated product. Many studies have investigated the laser alloying of titanium for improving wear resistance. Several have attempted to improve the wear resistance through the insitu synthesis of hard phases such as titanium nitride or titanium carbide through a laser alloying processes with nitrogen gas or carbon powder [6-10]. Laser alloying is flawed, however, as the brittleness of these hard phases often promotes the formation of cracks in the laser-alloyed zone.

Earlier our group developed a novel laser alloying technique with a light-transmitting resin as a source for the carbon element using a CW fiber laser [11]. The technique produces a crack-free laser-alloyed zone containing titanium carbide particles via a reaction between the molten titanium and the pyrolysis products of the resin at the interface of the resin and substrate. However, the problem of this technique is that the resin layer is easily damaged when the laser heat input become large. When we set the laser power as low as possible to avoid the damage of the resin layer, a laser-alloyed zone was not obtained because the melting of the titanium substrate was not occurred. A fast laser scanning speed and an adequate laser power for surface melting were required to form a laser-alloyed zone preventing the damage of the resin layer. While a fast laser scanning speed effectively averts this damage, it results in a low volume fraction of titanium carbide in the laser-alloyed zone. The melting and solidification processes during laser alloying treatment at fast scanning speeds are also problematic, as they degrade the surface integrity in the laser-irradiated area.

From the above, the laser alloying process with a light transmitting resin using a CW laser seems to be unstable. A high peak power is available by using a nanosecond pulse laser, therefore a nanosecond laser might be able to melt the surface of substrate even though the total heat input is lower than that of a CW laser.

In the present study we applied laser alloying treatment using a nanosecond pulse laser in an attempt to prevent damage to the resin layer by reducing the heat input and improve the surface integrity by minimizing the mass of the molten metal.

\section{Experimental procedure}

The specimens were cut into $25 \mathrm{~mm} \times 25 \mathrm{~mm}$ squares from 5-mm-thick commercial pure titanium plates (grade 


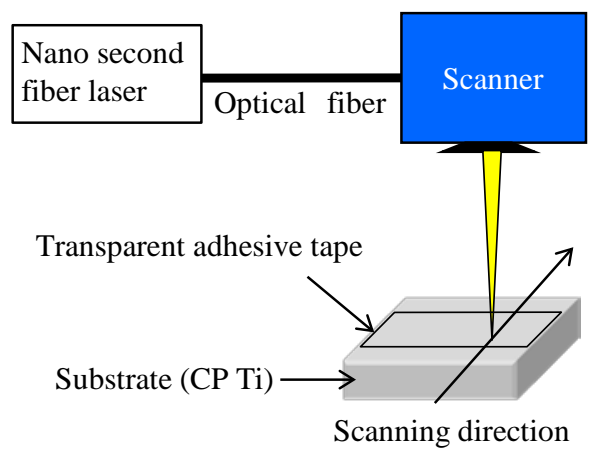

Fig. 1 Experimental setup.

Table1 Laser irradiation conditions.

\begin{tabular}{ll}
\hline Wave length & $1064 \mathrm{~nm}$ \\
Pulse width & $100 \mathrm{~ns}$ \\
Spot diameter & $30 \mu \mathrm{m}$ \\
Frequency & $200 \mathrm{kHz}$ \\
Average power & $10 \mathrm{~W}$ \\
Scanning speed & $100 \mathrm{~mm} / \mathrm{s}$ \\
Scanning pitch & $15 \mu \mathrm{m}$ \\
\hline
\end{tabular}

2), polished with SiC paper (P400), and cleaned with acetone.

Before commencing the laser irradiation, a commercial transparent adhesive tape consisting of cellulose acetate film and acrylic adhesive (total thickness of about $50 \mu \mathrm{m}$ ) was laminated on the surface of the titanium specimen as a light-transmitting resin.

A nanosecond single-mode fiber laser (YLP-1-100-2020) was focused to a spot diameter of $30 \mu \mathrm{m}$ and scanned across the specimen surface with a galvano scanner. Fig. 1 and Table 1 show the experimental setup and laser alloying conditions, respectively.

The microstructure and chemical composition of the laser-alloyed zone were studied using an optical microscope (OM), scanning electron microscope (SEM), and electron probe microanalyses (EPMA). X-Ray diffraction using $\mathrm{Cu} \mathrm{K} \alpha$ radiation was performed for phase identification.

The sliding wear properties of the laser-alloyed zone were evaluated with a ball-on-flat-type reciprocating wear tester using a 4.76-mm-diameter WC-Co ball as counterface material. The ball was ultrasonically cleaned with acetone and dried in air before it was mounted on the wear testing machine. The wear tests were performed without lubricants under the following conditions: temperature of $298 \mathrm{~K}$, relative humidity of $50 \%$, sliding speed of $20 \mathrm{~mm} / \mathrm{s}$, amplitude of $5 \mathrm{~mm}$, sliding distance of $72 \mathrm{~m}$, and load of $0.98 \mathrm{~N}$. The wear scars on the specimens and surfaces of the ball materials were investigated by SEM. A non-contact, three-dimensional measuring microscope was used to measure the depths of the wear scars.

\section{Results and Discussions}

\subsection{Surface appearance and microstructure}

Fig. 2 shows the appearance of the specimen after the laser alloying treatment. The surface of the titanium laser

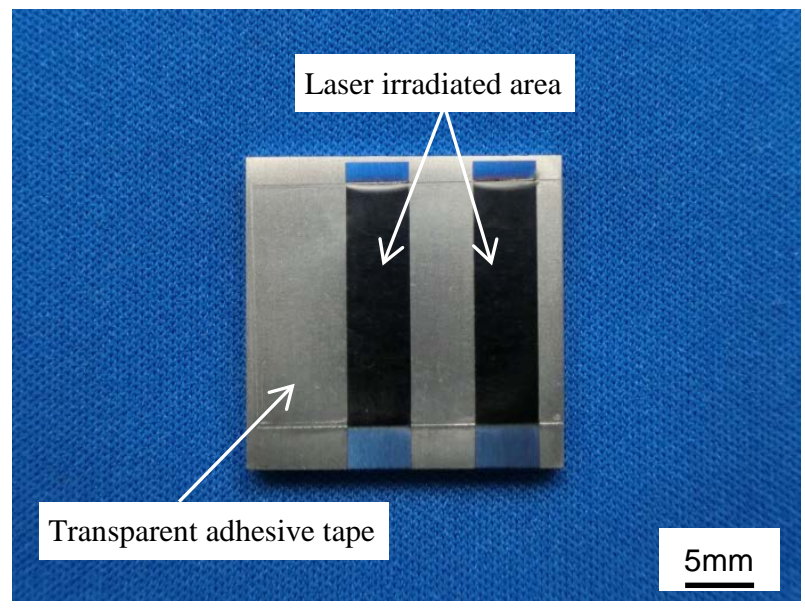

Fig.2 Appearance of the specimen after laser alloying.
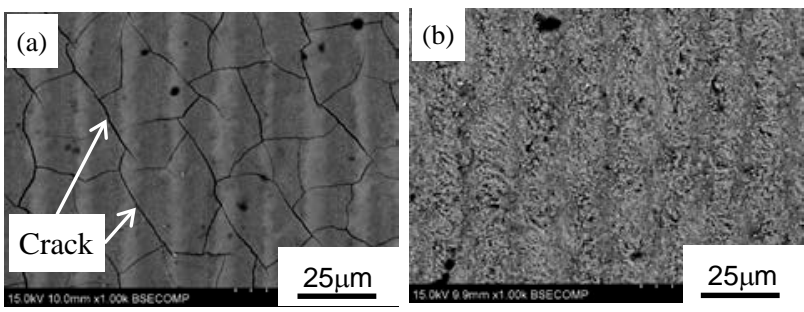

Fig.3 SEM images of the surface of the specimen.

(a)The area directly laser irradiated without the adhesive tape.

(b)The laser-alloyed zone with the adhesive tape.

irradiated through the adhesive tape was noticeably blackened. The surface irradiated without adhesive tape exhibited interference colors attributable to surface oxidation.

Under adequate laser conditions, the surface of the adhesive tape was not damaged due to the high laser transmittance of the adhesive tape. The thickness of the adhesive tape was thinner than the resin layer $(0.2-\mathrm{mm}-$ thick PMMA layer) we used in previous study [11]. However, the adhesive tape was not easily damaged by the nanosecond laser irradiation because the total heat input was lower than that of the CW laser.

The surface of the laser-alloyed zone was observed by scanning electron microscopy after removal of the adhesive tape and ultrasonic cleaning with acetone. Fig. 3 shows SEM images of the surface after laser irradiation. While no cracks were observed in the laser-alloyed zone (Fig. 3(b)), cracking was abundant in the area directly irradiated in air without the adhesive tape (Fig. 3(a)).

Fig. 4 (a) shows SEM images of the cross section of the specimen after laser alloying treatment. For comparison, Fig. 4 (b) shows the laser-alloyed zone formed by the CW laser in our previous study [11]. The thickness of the laseralloyed zone formed by the nanosecond laser was about 5 $10 \mu \mathrm{m}$, thinner than that of the CW laser. In the both laseralloyed zone, the second phase particles formed by the reaction between the molten titanium and pyrolysis products of the resin, were observed. In the laser-alloyed zone formed by the nanosecond laser, the second phase particles were almost uniformly distributed, but in the case of the CW laser, the second phase particles were segregated in the top surface of the laser-alloyed zone. It seems that high peak power of the nanosecond laser promote the 

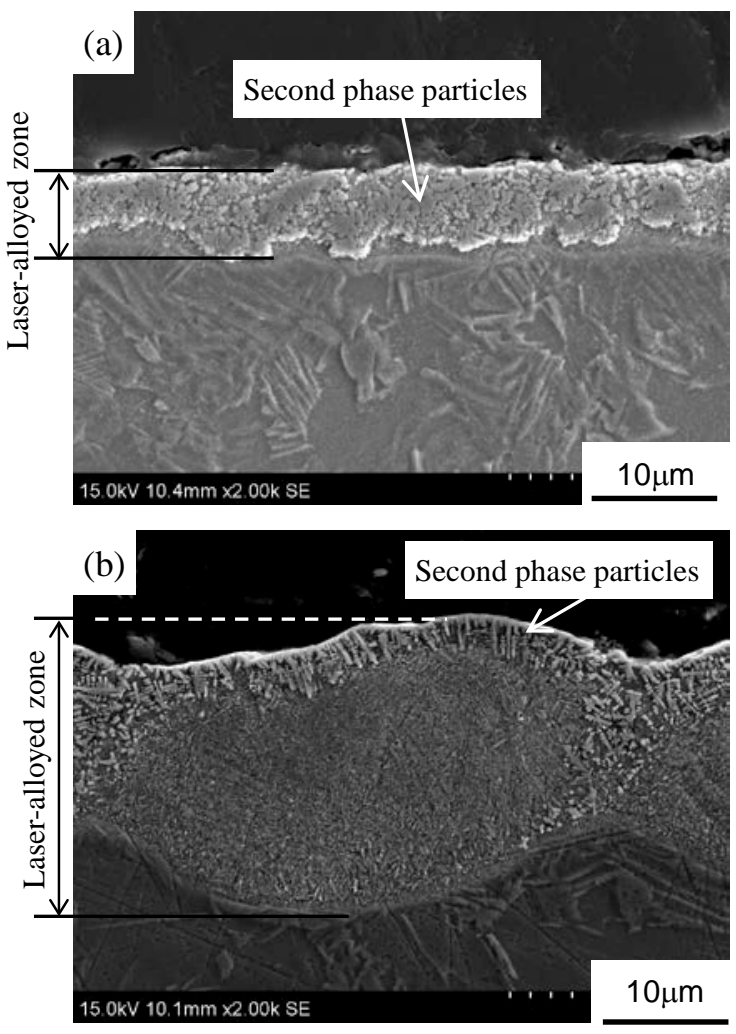

Fig.4 SEM images of the cross section of the specimen after laser alloying using (a) nanosecond laser (Average power $10 \mathrm{~W}$, Scanning speed $100 \mathrm{~mm} / \mathrm{s}$, Scanning pitch $15 \mu \mathrm{m}$ ) (b) CW laser (Power 30W, Scanning speed $150 \mathrm{~mm} / \mathrm{s}$, Scanning pitch $50 \mu \mathrm{m})$.

stirring the molten pool, consequently the homogeneous concentration of alloying elements were achieved.

To compare the surface integrity of each laser-alloyed zone, surface profiles were measured by 3D measuring system attached to SEM (Hitachi, 3D-View) (Fig. 5). The surface roughness (arithmetic mean roughness $\mathrm{Ra}$ ) of the laser-alloyed zone formed by the nanosecond laser was about $0.53 \mu \mathrm{m}$, while the value of the $\mathrm{CW}$ laser was about $2.36 \mu \mathrm{m}$. The surface roughness can be modified, if it is possible to reduce the scanning pitch. However, in the case of the CW laser, the resin layer was easily damaged due to large heat input, the laser-alloyed zone was not successfully obtained when the scanning pitch was reduced.

\subsection{Phase identification of the laser-alloyed zone}

Fig. 6 shows XRD results of the surfaces of the nonlaser irradiated titanium substrate and the laser-alloyed zone. In addition to the peak of the $\alpha$-titanium, a rock salt structure common to the TiC-TiN-TiO system was observed in the spectrum of the laser-alloyed zone.

The lattice parameter of this compound was calculated by using the diffraction peak corresponding to a (200) crystal plane, the diffraction angle $2 \theta$ was $40.23^{\circ}$. The calculated lattice parameter was $4.274 \AA$, a value relatively close to the lattice parameter of $\operatorname{TiN}(4.242 \AA)$. From the EPMA analysis, on the other hand, the nitrogen content of the laser-alloyed zone was found to be negligibly low. The adhesive tape contained no nitrogen as a composition
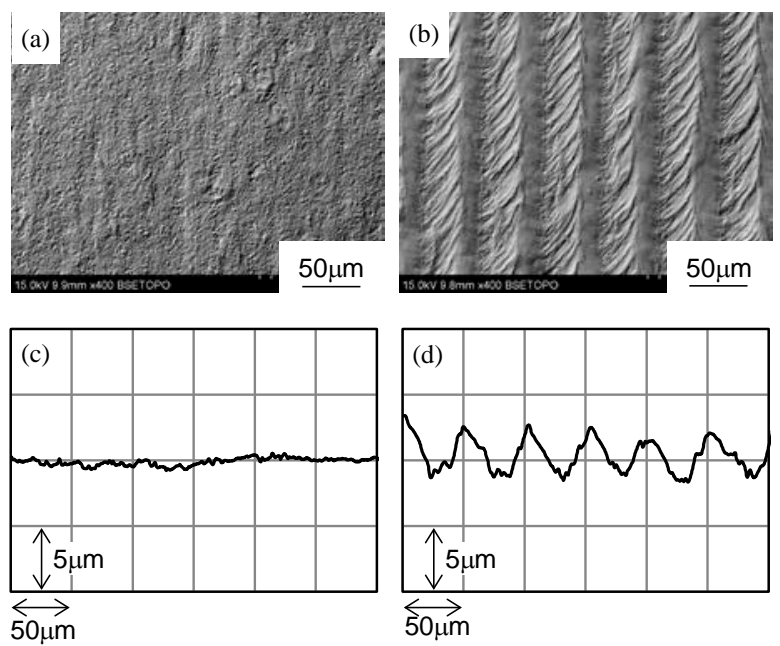

Fig.5 Surface topographic images ((a), (b)) and surface profiles ((c), (d)) of the laser-alloyed zone by SEM.

(a),(c) the laser-alloyed zone using nanosecond laser (Average power10W, Scanning speed $100 \mathrm{~mm} / \mathrm{s}$, Scanning pitch $15 \mu \mathrm{m}$ ) (b),(d) the laser-alloyed zone using CW laser (Power 30W, Scanning speed $150 \mathrm{~mm} / \mathrm{s}$, Scanning pitch $50 \mu \mathrm{m})$.
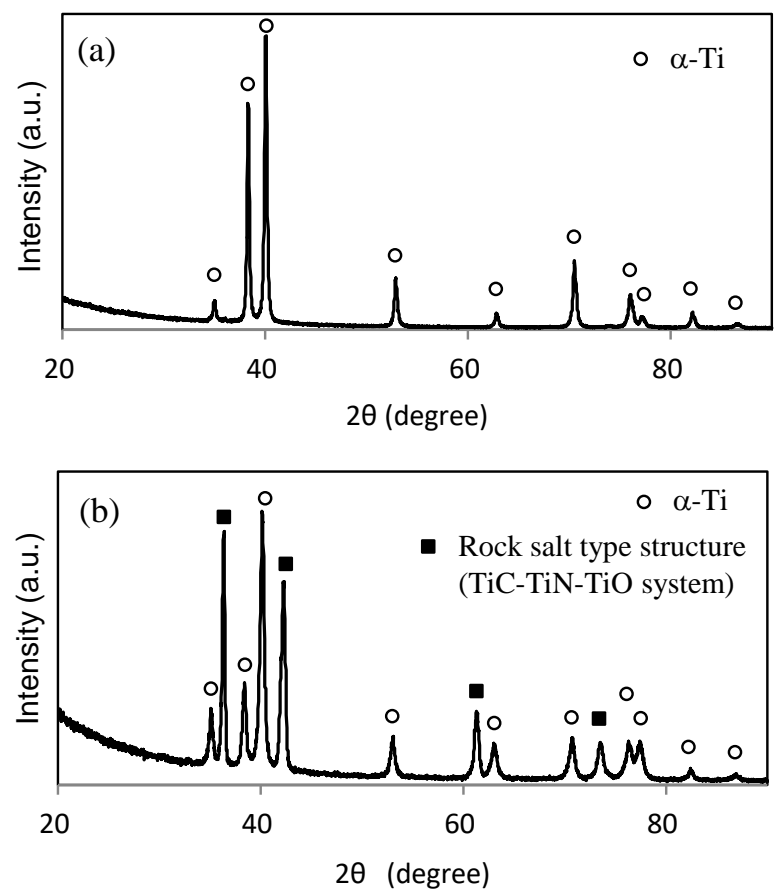

Fig.6 XRD patterns of (a) non-laser treated titanium substrate and (b) the laser-alloyed zone using nanosecond laser.

element, and the surface of the tape prevented the entrainment of nitrogen from air.

Fig. 7 shows the elementary distribution images by EPMA analysis. From these results, the second phase particles were found to be mainly composed of titanium and carbon. Slight amounts of oxygen were detected in the same area as the second phase particles. From these results, the second phase particles were identified as titanium oxycarbide. The XRD finding on the lattice parameter of the second phase particles, a value between TiC (4.327 $\AA$ ) and $\mathrm{TiO}(4.177 \AA)$, corresponded with this result. These 


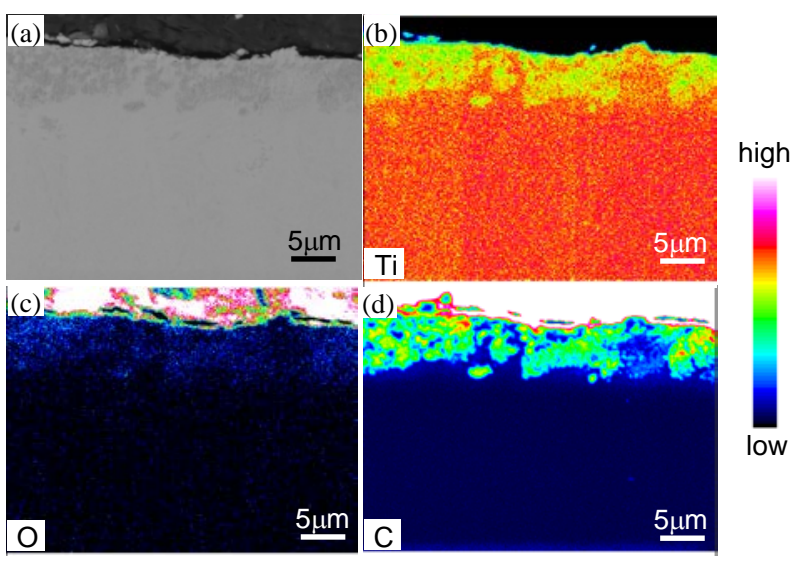

Fig.7 Results of EPMA plane analysis of the laser-alloyed zone using nanosecond laser.

(a) Secondary electron image (b) Titanium distribution image

(c) Oxygen distribution image (d) Carbon distribution image

carbon and oxygen elements were derived from the pyrolysis products of the acrylic adhesive. The laser irradiation induced the absorption of carbon and oxygen into the molten titanium, and the titanium oxycarbide formed during the subsequent solidification.

\subsection{Wear properties}

Fig. 8 shows the change of the friction coefficient at variable sliding distances. The friction coefficient of the non-laser treated titanium substrate ranged from about 0.5 to 0.8 , while that of the laser-alloyed zone was markedly lower, at 0.2 to 0.3 .

The adhesion and separation of the transferred particles induced sharp fluctuations in the friction coefficient of the non-laser-treated substrate to the WC-Co ball. The friction coefficient of the laser-alloyed zone, meanwhile, fluctuated much less than that of the titanium substrate.
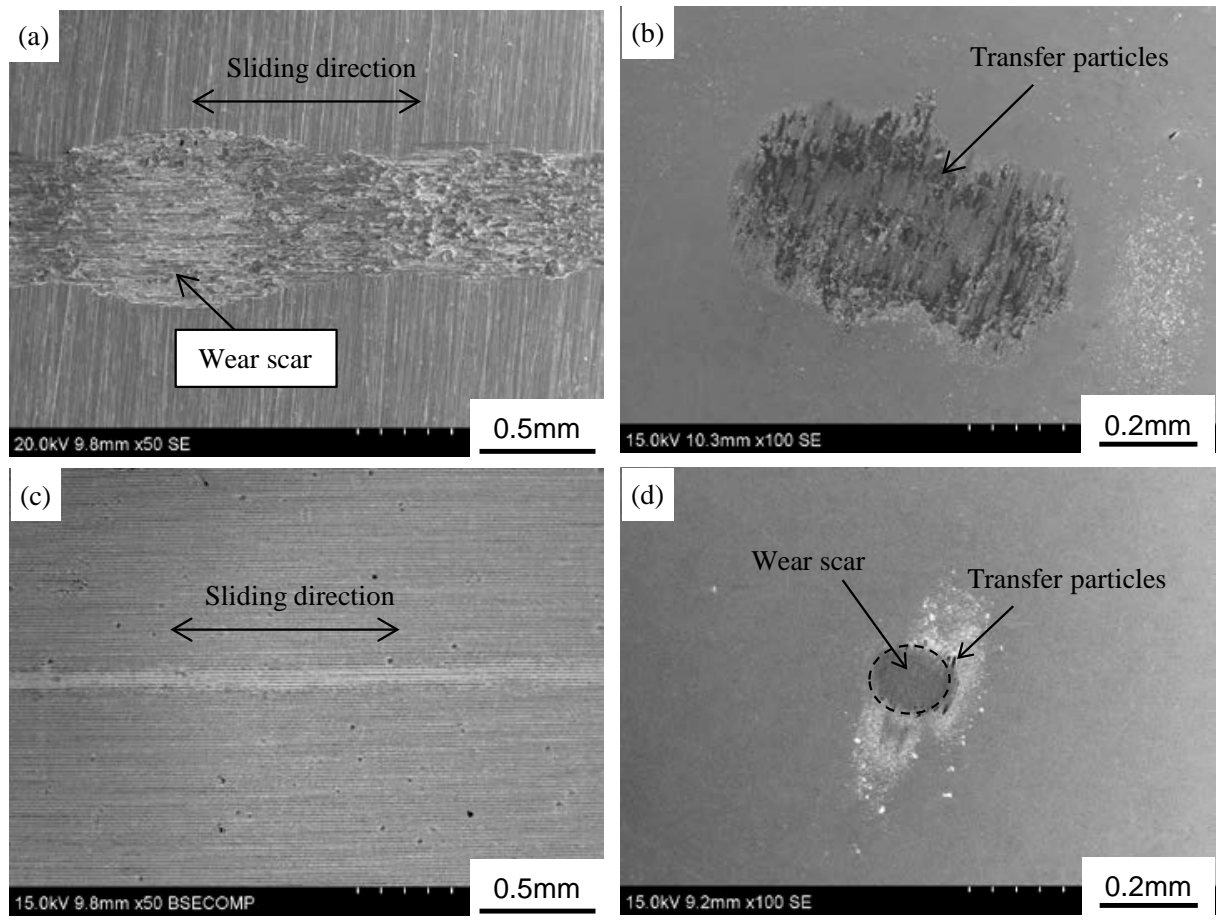

Fig.9 SEM images of the surface of the specimen ((a) non-laser treated substrate, (c) laser-alloyed zone) and the WC-Co ball after wear testing against non-laser treated substrate(b), and laser-alloyed zone(d).

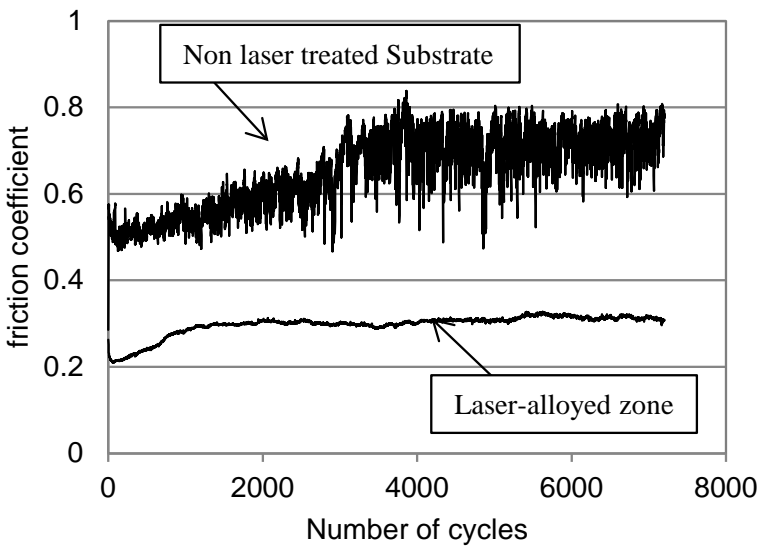

Fig.8 Friction coefficient during wear testing.

Fig. 9 shows SEM images of the specimen surfaces and the WC-Co ball counterfaces after wear testing. The wear scars on the non-laser-irradiated substrate were clearly observable and measured about $40 \mu \mathrm{m}$ in depth in the assessment by the non-contact three-dimensional measuring microscope (Fig. 9 (a)). In contrast, the wear scars in the laser-alloyed zone were too shallow to clearly see (Fig. 9 (c)). The SEM image of the WC-Co ball after wear testing against the non-laser-treated substrate revealed many transferred particles derived from the adhesion of titanium (Fig. 9 (b)). Few transferred particles, meanwhile, appeared on the surface of the WC-Co ball after wear testing against the laser-alloyed zone (Fig. 9 (d)). EDS analysis revealed that these transferred particles were titanium oxides. It is known that low-tensile strength materials such as titanium exhibit great material transfer to nonmetallic counterfaces [12]. The great affinity of titanium for oxygen results in the formation of an oxide surface layer, which is transferred to and adheres to counter 
materials. In the case of the laser-alloyed zone, the existence of titanium oxycarbide phase reduced the contact area of titanium and WC-Co, therefore adhesive wear was prevented.

The surface of the WC-Co ball was slightly worn after wear testing against the laser-alloyed zone (Fig.9 (d)). A. C. Fernandes reported that hardness of titanium oxycarbide decrease with increase oxygen content [13]. From the EPMA analysis, the X-ray intensity of oxygen was much lower than that of carbon (Fig.7), so the titanium oxycarbide obtained in this laser alloying process have higher hardness than WC-Co conterface material. As a result, the laser-alloyed zone was not abrasively worn by WC-Co. The laser-alloyed zone exhibited superior wear resistance.

\section{Conclusions}

Laser alloying of pure titanium using a nanosecond laser with a light-transmitting resin was carried out. A defect-free, laser-alloyed zone of about $5-10 \mu \mathrm{m}$ in thickness was obtained. The laser-alloyed zone formed with the nanosecond laser was both shallower and smoother than the laser-alloyed zone formed by the CW laser. High volume fractions of titanium oxycarbide particles were dispersed in the laser-alloyed zone. The laser-alloyed zone had a low friction coefficient against the WC-Co ball and superior wear resistance.

\section{Acknowledgements}

The authors would like to thank Dr. Yasuhiro Michiyama for assisting with the wear testing experiments and Dr. Tomotake Hirata for performing the EPMA analysis.

This work was supported by JSPS KAKENHI Grant Number JP15K18224.

\section{References}

[1] K. G. Budinski: Wear, 151, (1991) 203.

[2] S. Kameoka, S. Motonishi and H. Uchida: Surf. Coat. Tech., 169-170, (2003) 316.

[3] K. Nakata: J. Jpn. Weld. Soc., 63, (1994) 273.

[4] C. W. DRAPER and C. A. EWING: J. Mater. Sci., 19, (1984) 3815.

[5] W. M. Steen: Laser Material Processing third edition, (Springer, 2003) 227.

[6] H. M. Flower, A. Walker and D. R. F. West: Scripta Metallurgica, 19, (1985) 923.

[7] A. F. Saleh, J. H. Abboud and K. Y. Benyounis: Opt. Laser. Eng., 48, (2010) 257.

[8] V. M. Weerasinghe, D. R. F. West and J. de Damborenea: J. Mater. Proc. Technol., 58, (1996) 79.

[9] J. D. Majumdar: Physics Procedia, 12, (2011) 472.

[10]T. Yamaguchi, H. Hagino, M. Takemura and A. Nakahira: J. Jpn. Soc. Powder Powder Metall., 61, (2014) 481.

[11]T. Yamaguchi and H. Hagino: Opt. Laser. Eng., 88, (2017) 13.

[12] ASM Handbook Vol.18 (AMS International 1992) 778.

[13]A. C. Fernandes, P. Carvalho, F. Vaz, N. M. G. Parreira, P. Goudeau, E. L. Bourhis and J. Riviere: Plasma Process. Polym., 4, (2007) S83.

(Received: May 23, 2017, Accepted: September 30, 2017) 